\title{
Indonesia y su proceso de consolidación como potencia media bajo el período presidencial de Joko Widodo (2014-2017) (2017-2019)
}

Laura Daniela Molina Cortes*

\section{RESUMEN}

La multipolaridad del sistema internacional se ha visto reflejada por el surgimiento de nuevos poderes que buscan generar influencia y aspiran a cumplir un rol mucho más activo dentro del sistema internacional, socavando el dominio y posicionamiento de algunas potencias occidentales. Indonesia es un claro ejemplo debido a que desde su nacimiento pasó por diversos cambios, en donde experimentó varias crisis y tiempos prósperos que le ayudaron a crecer como Estado, y desde un inicio plantearse la idea de obtener un papel destacado a nivel mundial. En su proceso de poder consolidarse como potencia media ha implementado una clara estrategia diplomática, enfocada en demostrar su potencial, influencia y credibilidad frente a los demás. Esta estrategia hace alusión al Eje Marítimo Global, implementado por Joko Widodo, una visión marítima que ha destacado la posición geoestratégica de Indonesia, la cual la hace merecedora de grandes beneficios tanto económicos como comerciales. Además, ha incentivado el interés en participar activamente de los asuntos regionales haciéndole frente a diversos fenómenos, y la importancia de mantener buenas relaciones con sus vecinos y con las grandes potencias. Estos factores, sin lugar a duda, juegan un papel importante para reafirmar su rol e influencia dentro del sistema internacional. Sin embargo, existen dificultades de seguridad interna y externa que necesitan ser abordadas para que no se vuelvan un obstáculo dentro de su proceso de consolidación como potencia media (Pavez \& Rosales, 2016).

* Profesional en relaciones internacionales. Pontificia Universidad Javeriana. Bogotá, Colombia. [molina_laura@ javeriana.edu.co]; [oRCID https://orcid.org/0000-0002-4962-0900].

Recibido: 2 de diciembre de 2020 / Modificado: 11 de febrero de 2021 / Aceptado: 22 de febrero de 2021

Para citar este artículo:

Molina Cortes, L. D. (2021). Indonesia y su proceso de consolidación como potencia media bajo el período presidencial de Joko Widodo (2014-2017) (2017-2019). OASIS, 34, pp. 179-196

DOI: https://doi.org/10.18601/16577558.n34.10 
Palabras clave: Indonesia, potencia media, eje marítimo global, diplomacia marítima

\section{INDONESIA AND ITS CONSOLIDATION PROCESS AS A MIDDLE POWER IN THE GOVERNMENT OF JOKO WIDODO (2014-2017) (2017-2019)}

\section{ABSTRACT}

The multi-polar international system has reflected the emergence of new powers that want to influence and play an active role within the international arena, undermining the dominance of some Western powers. For instance, Indonesia has experienced different situations of crises and success that helped its rise as a State with a prominent worldwide role. In the process to continue its consolidation as a middle power, the country has implemented a diplomatic strategy, focused on showing its potential, influence, and credibility in relation to others. This strategy refers to the Global Maritime Axis, a maritime vision that has highlighted Indonesia's geostrategic position, as well as economic and commercial benefits, and which was implemented by Joko Widodo. Moreover, Indonesia has encouraged interest in participating in regional affairs by facing various phenomena, as well as the importance of maintaining good relationships among its neighbors and with the great powers. These factors play an important role because they state the role and influence within the international system. However, there are internal and external security issues that need to be addressed because represents an obstacle in its consolidation process as a middle power (Pavez \& Rosales, 2016).

Key words: Indonesia, middle power, global maritime axis, maritime diplomacy.

\section{INTRODUCCIÓN}

En el nuevo orden global, los Estados pertenecientes al sur global han comenzado a adquirir visibilidad debido a que han ido en ascenso, jugando un papel central no solo en la economía mundial, si no en diferentes asuntos de la agenda internacional. Estas sociedades han compartido una historia que ha sido marcada por el colonialismo e imperialismo que, de algún modo, las dejó marginadas, enfrentado dificultades para alcanzar sus objetivos económicos y políticos en la nueva modernidad, tanto capitalista como socialista. Sin embargo, resurgen con un nuevo discurso que evidencia un rol cada vez más visible en la agenda internacional, teniendo como resultado la reconfiguración de las relaciones de poder, en donde antiguos poderes han dejado de ser los únicos protagonistas, y se ha dado paso a aquellos que buscan sobresalir en la política mundial (Dirlik, 2007). Estos nuevos poderes emergentes pretenden alcanzar un reconocimiento en las principales esferas de influencia, en donde puedan adquirir otros roles y responsabilidades en la gobernanza global por medio de iniciativas multilaterales que les permitan mantener un buen estatus dentro del sistema (Chandra, 2018). Indonesia hace parte de estos Estados del Sur Global, ya que en su papel como potencia media ha manifestado desde siempre la importancia de tener un rol activo en los asun- 
tos globales, y a su vez reforzar su liderazgo en la región del sudeste asiático.

En primer lugar, dentro de este artículo de investigación será pertinente entender a qué hace referencia el concepto de potencia media para identificar si Indonesia cumple con los requisitos, y puede ser considerada con este estatus bajo el período de Joko Widodo. También, se realizará un pequeño estudio de contexto que refleje cómo este país ha venido trazando su camino como un poder medio, teniendo en cuenta las experiencias de prosperidad y crisis a lo largo de su historia. En segundo lugar, será relevante identificar en qué escenarios Indonesia cumple el rol de potencia media, por tal motivo se explicará cómo el Eje Marítimo Global es un elemento transcendental que puede reunir la esencia de este país como un poder medio. Además, se tendrá en cuenta el accionar del país dentro de la Asociación de la Cuenca del Océano Índico (Iora, por sus siglas en inglés), debido a que es una importante plataforma de cooperación regional que puede complementarse perfectamente con la identidad y los intereses de Indonesia. Y, por último, se identificará qué factores pueden obstaculizar el rol del país y pueden afectar directamente la doctrina marítima de Joko Widodo.

\section{METODOLOGÍA}

El método de investigación utilizado en este trabajo de investigación es el estudio de caso con un enfoque cualitativo. Esto debido a que permite estudiar, de manera amplia y detalla$\mathrm{da}$, un problema o inquietud que necesita ser abordado y analizado a profundidad. Además, el estudio de caso se presta para acudir a una extensa fuente de recursos de información y, de esta manera, obtener una descripción mucho más detallada del caso de estudio, reuniendo de manera cronológica cada aspecto que necesita ser analizado (Creswell, 2007). Conforme a esto, se acudió a diversas fuentes de información como artículos de investigación, libros, noticias, páginas web oficiales como el Ministerio de Relaciones Exteriores de la República de Indonesia, y la página oficial del Banco Mundial para la obtención de datos macroeconómicos como el PIB y el PIB per cápita. Todas estas fuentes de información fueron vitales para la recopilación de la información necesaria y, a su vez, realizar un estudio de contexto.

Este trabajo de investigación, como se planteó antes, es un estudio de caso que tiene como principal objeto de estudio la República de Indonesia, en donde se hizo énfasis en la administración del presidente Joko Widodo (2014-2017) (2017-2019). Aquí se partió de una pregunta de investigación, la cual hace referencia a: ¿Cómo Indonesia continúa consolidándose como potencia media bajo el período presidencial de Joko Widodo? Teniendo en cuenta la importancia de dar respuesta a la pregunta, fue necesario acudir al concepto de potencia media para poder guiar el análisis y entender bajo qué criterios un Estado es considerado un poder medio. Además, una vez definido el concepto se tuvo en cuenta cómo sería el rol de Indonesia como potencia media bajo la identificación de escenarios clave que se prestaron para abordar este trabajo de investigación. 


\section{OBJETIVO GENERAL}

- Explicar cómo Indonesia continúa consolidándose como potencia media bajo el período presidencial de Joko Widodo.

\section{OBJETIVOS ESPECÍFICOS}

- Entender qué es potencia media y cuáles son las características que la definen.

- Identificar en qué escenarios Indonesia cumple el estatus de potencia media.

- Identificar qué factores pueden obstaculizar su comportamiento como una potencia media.

\section{¿CÓMO DEFINIR UNA POTENCIA MEDIA?}

Para llevar a cabo un adecuado análisis acerca del proceso de consolidación de Indonesia como potencia media, y a su vez identificar en qué escenarios el país cumple con este papel, es de vital importancia entender a qué hace referencia este concepto dentro de la disciplina de las relaciones internacionales, y bajo qué premisas un Estado se puede identificar con este rol. Este término nació en medio de un debate que se cernía sobre el nuevo orden mundial posterior a la segunda guerra mundial, cuando para muchos fue de vital interés identificar cuál sería el comportamiento diplomático de aquellos Estados que estaban asumiendo un rol mucho más visible dentro del sistema internacional (Efstathopoulos, 2018). Conforme a esto, algunos académicos recomendaron que la manera adecuada de identificar una potencia media sería por medio de su comportamiento y el impacto que esto tendría en la gobernanza global. Por consiguiente, es primordial traer a colación algunas definiciones otorgadas por varios académicos que contribuyen a encontrar los criterios determinantes que diferencien las potencias medias de las grandes potencias.

De acuerdo con lo anterior, Según Emmers y Teo (2018)

We conceive of middle power as a status, or role that is salient in every situation. It is constituted by three auxiliary roles: being a good international citizen, a supporter of multilateralism; and a supporter of the existing international order. These auxiliary roles serve as the internal attributes of middle power status and exist in necessary and sufficient conditions to constitute this concept (p. 414).

Según, Thies y Sari (2018), "We define middle powers according to their material capabilities and identity. In other words, middle powers are those states that occupy a certain position in rankings of material resources and view themselves to be middle powers" (p. 10).

Por otro lado, dentro del concepto de potencia media se han desarrollado cinco enfoques y cada uno de ellos define la esencia y las características que debería tener un poder medio para su adecuada identificación. El primero de ellos es el enfoque identitario, el cual hace referencia a la importancia de que el Estado se auto identifique como potencia media, ya que esto va a trazar el camino de su comportamiento en el escenario internacional. Y a su vez, se necesita de una base sólida y coherente en su actuar para que los otros actores le otorguen ese reconocimiento. El enfoque jerárquico hace la distinción de los Estados que poseen una combinación de determina- 
dos recursos materiales con otros que no los tienen, y de acuerdo con esto se posicionan en una escala que mide su poder material. La capacidad militar, rango económico, nivel de desarrollo, población, tamaño del territorio y la posesión de ciertos recursos naturales son atributos primordiales que determinan el rango de estos Estados dentro del sistema internacional (Thies y Sari, 2018).

El enfoque conductual, plantea cuáles son los patrones de comportamiento que caracterizan a las verdaderas potencias medias. En primera instancia, estos Estados poseen una fuerte inclinación por participar y apoyar las iniciativas multilaterales, que los ayude a involucrarse en determinados asuntos globales que requieren atención. Según Cooper et al. (como se citó en Chapnick,1999) "their tendency to pursue multilateral solutions to international problems, their tendency to embrace compromise positions in international disputes, and their tendency to embrace notions of good international citizenship to guide their diplomacy" (p. 75). En segunda instancia, buscan comportarse como buenos ciudadanos internacionales para contribuir a la estabilidad y bienestar de la comunidad internacional, demostrando su responsabilidad para promover un entorno de mediación y buena comunicación entre los actores (Emmers y Teo, 2018). Y, por último, actúan por medio de la implementación de una "diplomacia de nicho", en la cual Behringer (como se citó en Kríž y Brajerčíková, 2019), planteó que, en este tipo de diplomacia, los Estados considerados poderes medios tienen la oportunidad de asumir un rol protagónico en cuestiones sociales, económicas y humanitarias donde las grandes potencias no están interesadas, o simplemente esto no hace parte de sus prioridades.

Por otro lado, el enfoque funcionalista señala que un poder medio es un actor que ayuda en el cumplimiento de ciertas funciones clave del sistema internacional y tiene la habilidad de contribuir a situaciones específicas, en donde el Estado tenga la confianza de actuar de acuerdo con sus capacidades y habilidades. Kríž y Brajerčíková (2019) plantean que un Estado considerado potencia media debe cumplir con determinadas funciones que ayuden a la estabilidad del sistema internacional, en donde es primordial poseer suficientes capacidades y habilidades para abordar determinadas áreas y así asumir un rol de responsabilidad. Un claro ejemplo, es la implementación de una diplomacia mediadora que ayude en la prevención y resolución de disputas o conflictos internacionales (Efstathopoulos, 2018). Y, por último, el enfoque sistémico se ve en la necesidad de identificar, de una manera más sólida y clara, un poder medio, ya que los demás enfoques si bien son esenciales en el proceso de identificación de este estatus, no son del todo suficientes para identificar un poder medio. Por esta razón, es indispensable medir el impacto y efecto que pueden tener las acciones de una potencia media sobre los otros actores, y en cómo se constituye el sistema internacional. Según Kríž y Brajerčíková (2019) "una potencia media es un Estado que es capaz de defender sus intereses nacionales vitales y al mismo tiempo influir significativamente en los principales parámetros del orden mundial existente" (p. 48). Este enfoque hace alusión a que las acciones emprendidas por los Estados deben tener cierto impacto sobre el sistema 
internacional y que esto logre influenciar a los demás actores que se encuentran a su alrededor, dado que necesitan ir construyendo un nivel de credibilidad que respalde su estatus de potencia media.

Como parte de esta investigación, es primordial realizar un breve estudio de contexto para dar cuenta de cómo ha sido la historia de este país, que ha estado marcada por diversos cambios, en donde ha experimentado tiempos de crisis y de prosperidad. Con el gobierno de Sukarno, Indonesia estuvo bajo la influencia de un líder comunista, el cual dirigió su mandato bajo un régimen antiliberal, alejándose totalmente de las premisas occidentales capitalistas y así defender su rechazo al colonialismo e imperialismo como parte de su posición en el sistema internacional. Durante este período la economía vivió grandes dificultades. El comercio exterior se vio afectado por la implementación de regulaciones restrictivas; la deuda externa creció; hubo un incremento de la inflación anual de más del 600\%; y como si no fuera poco se encontraba dentro del grupo de los países más pobres del mundo. Además, los problemas como la corrupción, políticas radicales y actitudes represivas dejaron como resultado la pérdida de confianza a nivel nacional e internacional (Coleman. et al., 2007).
Todo esto fue el detonante de que se llevara a cabo un golpe de Estado auspiciado por el general Suharto, el cual llegó al poder marcando un período crucial en distintos aspectos. Uno de ellos fue el nuevo direccionamiento que le dio a la política exterior del país, en donde cambió su postura hacia occidente; alivió las tensiones con Malasia y Kuala Lumpur; participó activamente en el movimiento de los no alineados; y enfatizó en la importancia de la Asean que pudiera impulsar el liderazgo regional del país. Sin embargo, este líder dejó que las fuerzas armadas tuvieran capacidad de decisión en diversos asuntos. Uno de ellos fue las relaciones con la República Popular de China, las cuales estuvieron congeladas la mayoría de su mandato. Y el otro asunto fue la decisión sobre la independencia de Timor Oriental en 1975, un episodio que marcó al país porque afectó su integridad territorial (Batabyal, 2002). Por otro lado, su gobierno fue clave en la transición hacia un país industrializado y desarrollado bajo un modelo económico totalmente diferente. Desde un principio tuvo como misión estabilizar y recuperar la economía por medio de algunas estrategias ${ }^{1}$ encaminadas hacia la apertura en el mercado internacional; incrementar la confianza en el mercado doméstico; adoptar políticas favorables para atraer la inversión extranjera ${ }^{2}$; aumentar las exportaciones y rea-

\footnotetext{
1 Estas estrategias estuvieron enfocadas en un presupuesto equilibrado, la rehabilitación de la infraestructura y desarrollo a nivel agrícola (Marks y Van Zanden, 2012).

2 Estas políticas fueron la Ley de inversión extranjera de 1967, la cual permitió generar vínculos con grandes compañías americanas y japonesas (Arndt, 1983).
} 
lizar grandes avances en la disminución de la pobreza (Marks y Van Zanden, 2012).

Desde los setentas hasta los ochentas, se vivió un verdadero crecimiento económico lleno de prosperidad gracias a los dos booms petroleros $^{3}$, que llevaron a que se diera un incremento en el precio del petróleo a nivel mundial. Todo esto trajo consigo un aumento en los ingresos del gobierno, los cuales fueron vitales para mejorar sus inversiones y ganancias (Arndt, 1983). Infortunadamente, a finales de los noventa se vivió un período sombrío con el impredecible declive de su economía debido a la caída en los precios del petróleo y la crisis financiera asiática en 1997, la cual dejó consecuencias devastadoras por las pérdidas y contracciones que llevaron al gobierno a acudir al Fondo Monetario Internacional (Marks y Van Zanden, 2012). Todos estos episodios desencadenaron el descontento por parte de la opinión pública, y alentó a que la población protestara en contra de un gobierno que, en su afán por lograr el crecimiento económico, ignoró aspectos políticos y sociales que afectaron la estabilidad del país, obligando al general Suharto a renunciar.

En los gobiernos siguientes de Habibie (1998-1999) y el de Abdurrah Wahid (1999-2001), se vivió un período de desestabilización y de seguridad interna que amenazó la imagen del país a nivel nacional e internacional, debido a las crisis separatistas en regiones como Papúa y Aceh, y la independencia oficial de Timor Oriental en 1999 que significó una grave pérdida. Esto ya venía dándose en la era de Suharto porque muchas de estas provincias se quejaron de problemas económicos, culturales y sociales debidos a la desigualdad y a las violaciones de derechos humanos. Tanto Habibie y Wahid buscaron dar una solución por medio de un proceso de descentralización y poder dar una autonomía especial a estos territorios y evitar una desintegración del archipiélago (Heiduk, 2014). Con respecto a la economía mostró signos de recuperación, la moneda se fortaleció, la inflación bajó y las exportaciones no petroleras mostraron signos de recuperación. Sin embargo, en estos dos gobiernos el problema de corrupción persistía afectando, de alguna forma, la estabilidad económica (Marks y Van Zanden, 2012). Más adelante, en el 2002, llegó al poder Megawati Soekarnoputri en medio de un sentimiento de incertidumbre hacia el comportamiento de la economía. A pesar de esto, la moneda logró estabilizarse, la inflación bajo relativamente y otros aspectos macroeconómicos mostraron lucidez (Smith, 2003). En cuanto a la política exterior, el país siguió dándole protagonismo a la Asean como una iniciativa crucial para mantener sus relaciones con la región y, a su vez, este organismo dio apoyo a la importancia de mantener la integridad territorial del archipiélago. Esto

\footnotetext{
3 El primer boom petrolero tuvo lugar en 1972-1973, en donde los miembros de la Opec acordaron cuadruplicar el precio del petróleo desde $\$ 3.70$ dólares por barril y $\$ 12.60$ dólares por barril para cada año respectivamente. El segundo boom petrolero tuvo lugar en 1979-1980 (Arndt, 1983).
} 
debido a que los miedos de nuevos procesos secesionistas siguieron presentes, en especial con Papúa, una región mayoritariamente cristiana que podía obtener el apoyo occidental (Batabyal, 2002).

Con la llegada de Susilo Bambang Yudhoyono (2004-2014) la economía creció satisfactoriamente, ya que alcanzó niveles nunca antes logrados desde el Nuevo Orden de Suharto. El 2011 significó prosperidad para el país porque tanto los índices de pobreza y desempleo descendieron; el pIB tuvo resultados positivos gracias al incremento en el consumo doméstico y se realizaron inversiones en infraestructura e industrias promovidas por locales y extranjeros (Mietzner, 2012). Para el 2014, último año de su mandato, se mostraron signos de debilidad en los índices macroeconómicos debido a que el рів bajó un $5.0 \%$, la rupia perdió su valor en un $25 \%$ y las exportaciones fueron menores $-2.2 \%$. que el año anterior. Todo esto fue resultado del colapso en el precio de productos importantes para el país como la palma de cera y el carbón, golpeando gravemente la economía (Mietzner, 2015). Yudhoyono fue un actor clave en la promoción de Indonesia como una creciente potencia media que buscaba tener un papel mucho más destacado en las relaciones internacionales (Emmers y Teo, 2018).

Bajo este mandato, Indonesia manifestó su interés en diferentes asuntos globales y en fortalecer sus relaciones con los demás Estados. Este comportamiento se vio reflejado en su participación en distintas organizaciones internacionales; su interés en abordar conflictos como el de Tailandia y Camboya (Mietzner, 2012). Y promover buenas relaciones bajo su lema miles de amigos y cero enemigos, en donde manifestó la importancia de mantener relaciones de amistad con todos los actores del sistema internacional, promoviendo la importancia de contar con el apoyo de las dos grandes potencias, en este caso de China y Estados Unidos. Estas acciones promovidas por el gobierno contribuyeron a darle mayor forma a un perfil diplomático que animaba al país a identificarse como una potencia y economía en ascenso (Connelly, 2014).

\section{INDONESIA, UNA POTENCIA MEDIA CON PROYECCIÓN MARÍTIMA}

Es indiscutible que un Estado catalogado como potencia media debe tener un grado de influencia e impacto dentro del sistema internacional, en donde sus intereses e identidades se vean representados y, de esta manera, identificar en qué escenarios puede destacarse y obtener credibilidad, de acuerdo con sus atributos materiales y no materiales. Por tal motivo, Indonesia como un Estado archipelágico y marítimo puede materializar su papel como potencia media porque es una herramienta que significa poder proyectarse en el escenario regional y global. Tal cual como se refleja en la doctrina de Jalesveva Jayamahe, la cual significa que "en el mar es donde triunfaremos" (Sambhi, 2015). Esto hace alusión a que el mar y los océanos son un claro escenario para que Indonesia tenga la confianza en desempeñar un rol mucho más creíble, en donde pueda interponer sus intereses como una verdadera potencia media.

En el 2014, con la llegada a la presidencia de Joko Widodo, más conocido como Jokowi, 
la política exterior ${ }^{4}$ ha estado orientada bajo el lema de Visi-Misi, siendo esta la esencia que determina las prioridades que debe tener el país a la hora de comportarse en el sistema internacional. Estas prioridades hacen alusión a promover la identidad de Indonesia como un Estado archipelágico y marítimo; resaltar el rol de Indonesia como una potencia media que se comprometa en abordar determinados asuntos internacionales y regionales; dar prioridad a los océanos Índico y Pacífico como parte de una estrategia de expansión regional, y priorizar la cooperación marítima a través de iniciativas multilaterales como la Asociación de la Cuenca del océano Índico (Iora) (Weatherbee, 2016).

En uno de sus primeros discursos como presidente, anunció el pilar fundamental de su política exterior llamado el Eje Marítimo Global, el cual está diseñado para fortalecer la seguridad, estabilidad, infraestructura y prosperidad económica del país. De acuerdo con Widodo, dentro de esta política se prevé desarrollar cinco objetivos clave que son: reconocer que la identidad de Indonesia es la de un Estado archipelágico y marítimo, que posee más de 1.700 islas, y está rodeada por los océanos Índico y Pacífico; fomentar la protección de los recursos naturales de la Zona Económica Exclusiva, con un enfoque en la soberanía marítima alimentaria; dar prioridad al desarrollo de la infraestructura marítima y a la conectividad tanto nacional, regional e internacional; promover una diplomacia marítima que invite a otras naciones a cooperar en distintos ámbitos y así eliminar los conflictos, la pesca ilegal, violaciones de la soberanía, la piratería y la polución marina, y desarrollar una política sólida de defensa marítima como responsabilidad para mantener la seguridad y protección de esta zona (Witular, 2014). Esto refleja, que el gobierno ha sido consciente de trazar un camino para que Indonesia pueda proyectarse como una potencia media, con una visión marítima mucho más sólida, enfatizando en su posición geoestratégica, la cual la hace merecedora de grandes beneficios.

Para llevar a cabo un adecuado análisis, es primordial entender que este estatus debe contar con una significativa posesión de recursos materiales para adquirir un rango de poder en el escenario internacional y, sobre todo, darle la suficiente confianza para actuar en determina-

4 El Ministerio de Relaciones Exteriores de la República de Indonesia. Los principales propósitos de la política exterior del gobierno de Joko Widodo van encaminados en fortalecer sus relaciones de cooperación con las regiones de Asia-Pacifico, África, América y Europa; continuar manifestando su papel de liderazgo dentro de iniciativas multilaterales como la Asean; participar activamente en temas de vital importancia para la comunidad internacional como la paz, seguridad, protección de los derechos humanos, desarrollo económico, socio-cultural, financiero, ambiental, comercial, industrial y de inversión por medio del fortalecimiento de la cooperación regional y multilateral; promover una imagen positiva del país; mejorar la diplomacia del país a través de los acuerdos internacionales; protección de la población indonesia en el exterior y, por último, mejorar la calidad de la política exterior, haciéndola más efectiva y avanzando conjuntamente con los intereses nacionales (Ministry of Foreign Affairs of the Republic of Indonesia, 2019). 
dos asuntos. En este orden de ideas, Indonesia con su posición geográfica cuenta con enormes ventajas y beneficios que son esenciales para su rol como potencia media en el ámbito económico (Nayanthara, 2016). Con la posesión de recursos naturales como el petróleo ${ }^{5}$, gas, minerales y aceite de palma, es evidente que esto ha representado un gran atractivo para la economía mundial (Alcaide, 2020). También, su ubicación es clave para el comercio internacional debido a que esto contribuye a estrechar lazos con importantes socios comerciales. En este sentido, para el país siempre ha sido vital tener un buen desempeño en su economía para obtener la posición de una de las economías más importantes y prometedoras en la región del sudeste asiático. De acuerdo con el Banco Mundial, Indonesia tiene un crecimiento anual en su PIB del $5.0 \%$ y en su PIB per cápita del 3.9\%. Por otra parte, es la décima economía más grande del mundo en términos de paridad de poder adquisitivo, en donde recientemente obtuvo el estatus de un país con ingreso medio alto. Además, gracias a su plan de desarrollo ha podido incrementar su competitividad en el mercado mundial; mejorar el capital humano y lograr importantes avances en la reducción de la pobreza con un $9.78 \%$ (The World Bank, 2020). Es por esto que esta doctrina puede ofrecer innumerables oportunidades para la economía y comercio marítimo, porque hace al país mucho más competitivo, aprovechando la oportunidad de conservar sus principales aliados comerciales y, a su vez, diversificar sus relaciones.

Por otro lado, La visión marítima promovida por el Eje Marítimo Global está directamente conectada en fortalecer el avance de Indonesia en términos comerciales, mejorar la conectividad entre todas sus islas por medio del desarrollo en infraestructura y proteger sus recursos marítimos (Sambhi, 2015). No obstante, hay que recalcar que el país no cuenta con la suficiente calidad en infraestructura y muchos de sus puertos han sufrido la falta de mantenimiento, impidiendo la conectividad a nivel interno y externo. De ahí que parte de su territorio quede rezagado de la economía nacional y no se pueda desarrollar todo su potencial (Nayanthara, 2016).

Pero, a pesar de esto, Jokowi le ha dado prioridad al desarrollo en la infraestructura, por esta razón, se busca llevar a cabo ambiciosos proyectos a lo largo del archipiélago, como grandes carreteras y puertos marítimos (Negara, 2016). Por lo tanto, con la política oceánica de Indonesia ${ }^{6}$, se quiere perseguir una proyección marítima a gran escala, y junto a ella llevar a cabo la iniciativa Sea Troll Road que busca construir 24 puertos marítimos con el

\footnotetext{
5 Haciendo una revisión al pasado, parte del crecimiento económico de Indonesia fue gracias a la posesión de recursos petroleros (Alcaide, 2020).

6 Esta política consta de 7 pilares fundamentales, los cuales hacen referencia a: impulsar un adecuado desarrollo de los recursos marinos; seguridad marítima; promover una gobernanza coherente en este campo; desarrollo de la economía marítima; gestión y protección marina; cultura marítima y, por último, diplomacia marítima (Lee, 2018).
} 
fin de convertir al país en un punto importante para el comercio internacional en las regiones del Asean y el indo-pacífico. Estos ambiciosos programas y proyectos necesitan ser financiados. Y es por esto que el presidente ha mostrado la necesidad de atraer inversión extranjera para la consecución de estos intereses, de hecho, China ha sido su principal socio y financiador, al igual que el Banco Asiático de Desarrollo de Infraestructura (АІІв) (Lee, 2018). Evidentemente, estos proyectos han sido vitales para el desarrollo del país y su economía, incrementando la competitividad a nivel internacional gracias a que se ha reconocido todo lo que puede ofrecer su posición geoestratégica y su visión marítima. De ahí la importancia de la infraestructura para prosperar económica y socialmente en un país que necesita implementar una visión que logre unir e integrar a todas sus islas por igual.

Indonesia con su proyección de Eje Marítimo Global representa una pieza fundamental para asumir un rol de liderazgo regional mucho más consistente, siendo esto un claro patrón de comportamiento de una potencia media. Es decir que, con esta doctrina, el país puede cohesionar sus intereses a la Asociación de la Cuenca del Océano Índico (Iora), la cual resulta ser una plataforma multilateral clave que aborda diversas coyunturas que involucran a toda una región. Y de esta forma, contribuir a la estabilidad, seguridad y bienestar para promover de una manera eficaz sus propósitos de darle prioridad al buen uso de los océanos y sus fronteras. Todo esto con el fin de expandir su influencia y generar un verdadero impacto que le otorgue credibilidad como una potencia media en ascenso.
En primer lugar, es importante recalcar que tanto Indonesia como los demás países que comparten fronteras con el océano Índico han estado expuestos a constantes amenazas que afectan su soberanía y seguridad. La explotación ilegal de los recursos, la pesca, el contrabando, el terrorismo y las controversias limítrofes han afectado la estabilidad de la región (Setiyanto, 2017). Y lastimosamente, han dado paso a que la desconfianza, y la falta de voluntad política de algunos Estados para afrontar estos problemas prime y se haga presente (Nayanthara, 2016). Es por esto, que se requiere de la cooperación multilateral para enfrentar estos desafíos que aquejan a toda una región. Según Sakhuja (como se citó en Setiyanto, 2017) "an openregionalism is needed, and its form of cooperation should be supportive, mutually complementary, and non- competitive" (p. 17). Indonesia, con su identidad marítima tiene la responsabilidad de promover soluciones que beneficien no solo a la región como tal, sino también al adecuado uso y manejo de los océanos Índico y Pacífico. Por lo tanto, el mejor escenario es participar activamente en iniciativas regionales como el Iora, en donde como prioridad en la agenda se subraye la unidad regional.

En este orden de ideas, el océano Índico cuenta con una gran importancia para Indonesia en su búsqueda de fortalecer la cooperación marítima en todo sentido, y poder desarrollar su doctrina como una herramienta diplomática para estrechar lazos con sus vecinos de la región y evitar la supremacía en esta zona (Setiyanto, 2017). En virtud de ello, la Asociación de la 
Cuenca del Océano Indico ${ }^{7}$ es una iniciativa regional que se creó para abordar todas las amenazas, y asuntos internacionales de gran relevancia. Esta organización se basa en cinco pilares fundamentales los cuales hacen alusión a: la seguridad y protección marítima; comercio; gestión pesquera; gestión de los desastres naturales; cooperación académica en ciencia y tecnología; intercambio cultural y promoción del turismo (Ministry of Foreign Affairs of the Republic of Indonesia, 2019). Parte de todos estos pilares se pueden ver reflejados en la doctrina marítima de Jokowi, evidenciando que hay una conexión clara y que el país ha acertado al ser parte de esta Asociación.

Para demostrar su capacidad de liderazgo, Indonesia tuvo la oportunidad de asumir la presidencia del Iora en los años 2015-2017, en donde el tema principal a desarrollar fue $e l$ fortalecimiento de la cooperación maritima para un océano Índico pacífico, estable y próspero. Además, fue anfitriona de la cumbre celebrada en el 2017, la cual juntó a los países miembros y se prestó especial atención a los temas relacionados con la seguridad marítima, asistencia humanitaria a posibles desastres, manejo de la pesca, comercio, inversión y turismo. Al mismo tiempo, se emitieron dos documentos de gran relevancia como el Acuerdo de Yakarta y la Declaración Conjunta de la Comunidad Empresarial Iora, clave para un crecimiento económico sostenible y equitativo. Y, también, adoptaron el Plan de Acción Iora y la Declaración para Prevenir y Combatir el Terrorismo y el Extremismo Violento (GK Today, 2017). Adicionalmente, en su rol como presidente se comprometió en realizar ciertos programas y actividades dirigidos a fomentar el diálogo en distintos ámbitos. Entre ellos están: el tercer diálogo del océano Índico; el simposio internacional del $20^{\circ}$ aniversario de Iora; el Centro de Innovación Empresarial Iora (BIC); la Guía de Inversiones de Iora; la 2a Conferencia sobre Economía Azul; el Taller regional sobre la intersección de cultura y civilización en el océano Índico; y la Exposición Cultural Iorag (Ministry of Foreign Affairs of the Republic of Indonesia, 2019).

$\mathrm{Al}$ considerar que Indonesia, en su rol de potencia media, tiene la misión de generar un verdadero impacto en la región, es innegable no destacar que el Iora es un escenario clave. Esto, debido a que puede fortalecer su proyección como un poder marítimo. De acuerdo con Carr (2014) "a systemic impact approach defines middle powers through their ability to alter or affect specific elements of the international system in which they find themselves" (p. 79). En efecto, el rol que ha tomado Indonesia ha afectado de algún modo el comportamiento de los Estados de la región, porque los ha influenciado a tomar consciencia

\footnotetext{
7 Creada en 1997. De ella hacen parte 21 países miembros: Sudáfrica, Australia, Bangladés, Comoras, India, Indonesia, Irán, Kenia, Madagascar, Malasia, Mauricio, Mozambique, Omán, Emiratos Árabes Unidos, Seychelles, Singapur, Somalia, Sri Lanka, Tanzania, Tailandia y Yemen. Y cuenta con algunos socios en cooperación como: Estados Unidos, Gran Bretaña, Japón, Alemania, Egipto, Francia y la República Popular China (Ministry of Foreign Affairs of the Republic of Indonesia, 2019).
} 
de los problemas y amenazas que enfrentan en común. Y como resultado, estos países han apoyado y aprobado el Acuerdo de Yakarta, en el cual se incorporaron los objetivos promulgados en el Eje Marítimo Global y, a su vez, los 21 miembros se comprometieron a: contribuir a la estabilidad, seguridad y respeto por la soberanía; cooperar en asuntos comerciales y de inversión; promover una pesca responsable y sostenible; mejorar el manejo en el riesgo de desastres; fortalecer la cooperación en ciencia y tecnología; fomentar el intercambio turístico y cultural; expandir la colaboración con otros países relevantes para la región y fortalecer el rol y las instituciones de la Asociación (IORA International, 2017).

Por otro lado, uno de los propósitos del Eje Marítimo Global es desarrollar una defensa marítima y el gobierno ha sido consciente de la importancia de fortalecer la capacidad del Estado en temas militares, con el fin de proteger las aguas indonesias, y de esta manera desarrollar una guardia costera completamente eficaz. La pesca ilegal, la explotación de los recursos marítimos y la violación a su soberanía son desafíos que requieren de una atención adecuada y la mejor manera es invertir y desarrollar eficazmente sus capacidades militares (Sambhi, 2015). Es por esta razón que las instituciones encargadas de la seguridad y defensa del país jueguen un papel crucial para hacerle frente a estas amenazas, de hecho, Widodo ha sido consciente que la Junta Nacional de Seguridad Marítima (Bakamla) y las Fuerzas Armadas (TNI) necesitan de la modernización en barcos, aviones de combate, buques de guerra y submarinos para defender al país (Sambhi, 2015). Como parte del compromiso de elimi- nar la pesca ilegal para cuidar el bienestar de los océanos, Joko Widodo ha implementado una estrategia firme y dura en contra de barcos extranjeros que pasan por sus aguas, de hecho el país ha hundido barcos provenientes de países como Vietnam, Filipinas y Malasia. Esto lo ha llevado a cabo porque cuenta con un respaldo legal para hacerlo por medio de la Convención de las Naciones Unidas sobre el Derecho del Mar (Nur, 2019).

Cabe considerar que Indonesia, a diferencia de las grandes potencias, cuenta con recursos limitados y su capacidad militar es uno de ellos, por ende, siempre va a buscar crear coaliciones con Estados que tengan potencial en la materia. Con Estados Unidos se tienen acuerdos de cooperación militar cada vez más amplios y profundos debido a que se cuenta con programas educativos, de capacitación y ejercicio conjunto, en donde las Fuerzas Armadas han estado totalmente involucradas, gozando de un mayor intercambio de conocimientos en el área militar. También, el país ha adquirido aviones de combate, semirremolques y algunos submarinos por parte de Corea del Sur (Weatherbee, 2016). Asimismo, con Rusia se han llevado importantes acuerdos para el suministro de aviones llamados Caza rusos Sukhoi-Su 35 de quinta generación, los cuales son capaces de cargar bombas y misiles para una defensa efectiva (Sputnik Mundo, 2018). En definitiva, en su deseo de contrarrestar las limitaciones que tienen sus capacidades militares que la hacen vulnerable a todas esas amenazas que están presentes, se han hecho importantes esfuerzos por modernizar su aparato militar todo en vista de reforzar su seguridad y demostrar que para el país no 
es un juego la protección de su soberanía en distintos aspectos.

\section{DESAFÍOS EN LA PROYECCIÓN MARÍTIMA DE INDONESIA}

El análisis procedente, comprende la necesidad de tener en cuenta que el sistema internacional está en constante cambio por el surgimiento de nuevos fenómenos. Y como consecuencia, los poderes medios pueden experimentar períodos de inestabilidad para poder mantener esta posición, por esta razón este rol necesita repensarse continuamente.

De acuerdo con lo anterior, Según Cox (como se citó en Carr, 2014)

the middle-power role is not a fixed universal but something that has to be rethought continually in the context of the changing system [...]. This claim is a reminder that any assessment of middle power status must be heavily driven by the context, as a relational understanding of power encourages (p. 78).

En este orden de ideas, es necesario considerar que el comportamiento de las potencias medias no es constante, dado que existen factores que pueden obstaculizar y opacar la estabilidad de este estatus. Como se ha venido planteando, Indonesia ha experimentado amenazas hacia su seguridad tanto internas y externas. Sin embargo, la controversia por el mar del Sur de China y las amenazas de secesión que enfrenta el país son problemas de gran trascendencia que si no se afrontan de la manera correcta pueden afectar el progreso del Eje Marítimo Global.

La controversia por el mar del Sur de China $^{8}$ con Indonesia surge a raíz de varios factores. En primer lugar, no existe una delimitación clara y definida de las fronteras; hay una competencia excesiva por los recursos marítimos de esta zona; se reclaman los derechos de pesca, los cuales no están legalizados. Además, la integridad de la Zona Económica Exclusiva está siendo amenazada por la incursión ilegal de varios países. Esto se puede ver reflejado en las tensiones que se han venido dando en las islas Natuna, ubicadas en la provincia de Riau, las cuales se encuentran dentro de la zona que reclama la República Popular de China (Sambhi, 2015). Adicionalmente, este territorio es clave para el transporte marítimo comercial del mundo, y cuenta con recursos como el petróleo y gas que son apetecidos por todos los países de la región. Es por esta razón, que la República Popular de China reclama casi el 90\% del área total que supuestamente está definida por la línea de los nueve trazos (Zorlu y Alam, 2019). Esta delimitación promulgada no cuenta con una base legal acorde con el derecho internacional, y con lo estipulado por la Convención de las Naciones Unidas sobre el Derecho al Mar, motivo por el cual Indonesia no acepta esta afirmación por parte del gigante asiático.

Indonesia se encuentra en una encrucijada porque ha tratado de manejar estas tensio-

8 El mar del Sur de China tiene limitaciones con Brunei, Camboya, China, Indonesia, Malasia, Filipinas, Singapur, Taiwán, Tailandia y Vietnam (Zorlu y Alam, 2019). 
nes de una manera prudente y diplomática con China, de hecho, ha sido mediadora en otras disputas abiertas con otros países como Filipinas, apoyando a que se dé una decisión por parte del Tribunal de Arbitraje para otorgarle derechos soberanos a este país (Nayanthara, 2016). Sin embargo, la escalada de tensiones es diaria porque continuamente pasan barcos pesqueros de forma ilegal, pero Indonesia ha sido muy clara en aplicar su política "terapia de choque" para enfrentar y destruir este tipo de embarcaciones (Sambhi, 2015). Sin lugar a dudas, esto puede causar un enfrentamiento mucho mayor y afectar lo que se quiere lograr con el Eje Marítimo Global porque la República Popular de China ha sido un socio potencial.

Por otro lado, ha estado muy latente la creciente amenaza por parte de los movimientos separatistas en algunas regiones de Indonesia que impacta negativamente el ideal de una unidad nacional promulgado por Pancasila ${ }^{9} \mathrm{y}$, a su vez, con la visión marítima de Joko Widodo porque cuestiona la identidad de Indonesia, reflejando que a pesar de las diferencias culturales no se ha dado una integración igualitaria para todas las islas. El país siempre ha vivido con este temor desde la independencia de Timor Oriental en 1997 que, de algún modo, dio paso a que otras regiones sintieran el deseo de no ser parte del archipiélago (Tiwon, 2000). Un claro caso es el de Papúa Occidental, al que el presidente ha prestado especial atención, debido a que se han dado movimientos armados que han contribuido a la desestabilización de la seguridad. Como un pequeño contexto, este territorio estuvo bajo el control holandés, pero en los sesentas fue entregado a la Autoridad Ejecutiva Temporal de las Naciones Unidas y después de un tiempo se decidió que fuera parte oficial de la isla. Indonesia para proteger esta zona contó con la presencia de sus militares y aplicó las leyes y normas del país. Sin embargo, estos actos ocasionaron políticas opresivas a los grupos étnicos que viven en Papúa y se les negó la oportunidad de la autodeterminación, y como resultado muchos fueron asesinados. Debido a esto se estableció el Movimiento Papúa Libre para oponerse a Indonesia y crear un solo Estado (Monineath, 2013).

Joko Widodo ha sido consciente de la importancia estratégica que tiene esta región para el país, porque es una de las provincias más ricas en recursos naturales, y su ubicación es valiosa para implementar actividades de vigilancia frente a la intrusión extranjera de barcos extranjeros en el Pacífico y las actividades de pesca ilegal. Y es por esto que ha tratado de implementar estrategias que generen el bienestar en la región por medio del desarrollo en infraestructura para fortalecer la conectividad y que haga parte de las Zonas Económicas Exclusivas (Syailendra, 2015). Sin embargo, las tensiones entre los papúes y el gobierno siempre han estado presentes. En el 2017 se realizó una petición al Comité de Descolonización de la ONU para solicitar la independencia; en el 2019

\footnotetext{
9 Pancasila se encuentra en la constitución de la República de Indonesia, la cual se basa en cinco principios que
} incluyen la creencia en un Dios, el humanitarismo, la unidad nacional, democracia y justicia social (Iskandar, 2016). 
se vivió un período bastante sombrío para el gobierno por el ataque racista hacia unos estudiantes provenientes de esta región, desatando protestas aún más violentas y como respuesta el país envió su equipo militar (https://www. bbc.com). Pareciera ser que el gobierno no ha prestado la suficiente atención a este problema de seguridad interna, si bien, ha implementado ciertos programas para la población de Papúa Occidental. Esto no ha sido suficiente, ya que se enfrenta a un problema en donde la religión y la cultura cumplen un papel fundamental, haciendo más difícil una solución duradera.

\section{CONCLUSIONES}

En definitiva, se puede establecer que Indonesia continúa consolidándose como una potencia media gracias a su proyección marítima, reflejada en el Eje Marítimo Global implementado bajo el período presidencial de Joko Widodo. Esto debido a que aspectos importantes planteados por el concepto de potencia media se ven integrados en esta visión. Gracias a su posición geoestratégica, el país posee recursos de gran importancia y es un punto clave para las actividades comerciales y económicas del mundo. Esto la ha beneficiado enormemente para reforzar su rol como una potencia media en el ámbito económico.

Por otro lado, se han llevado a cabo proyectos de infraestructura para promover la conectividad en sus islas, esto con la ayuda de grandes potencias como la República Popular de China. También, se le ha dado importancia a desarrollar una defensa marítima, que responda adecuadamente a las amenazas que aquejan al país. Además, la importancia que ha tenido el Iora para Indonesia es muy clara, debido a que se han logrado cohesionar los intereses del país para abordar ciertos asuntos relevantes, generando un verdadero impacto en la región.

No obstante, desafíos como la controversia por el mar del Sur de China y el conflicto separatista de Papúa Occidental pueden obstaculizar y opacar no solo su proyección marítima sino también su papel como potencia media. Esto porque, en el primer problema, China está directamente involucrada y parte de sus programas y proyectos de cooperación se pueden ver afectados. Además, si parte de su Zona Económica Exclusiva se ve amenazada de ser separada de Indonesia, significaría una enorme pérdida no solo a nivel económico, también un golpe a su identidad marítima.

\section{REFERENCIAS}

Acharya, A. (2014). Indonesia Matters: Asia's Emerging Democratic Power. Singapore. World Scientific Publishing Co.

Alcaide, J. (2020). El avance de la economía indonesia: contexto, aspectos positivos y desafios. Real Instituto Elcano.

Arndt, H. (1983). Oil and the Indonesian economy. Southeast Asian Affairs, 136-150. http:// www.jstor.org/stable/27908478The World Bank. (2020). The World Bank in Indonesia: Overview. Retrieved from https://www.worldbank.org/en/ country/indonesia/overview\#1

Batabyal, A. (2002). Change and Continuity in Indonesian Foreign Policy: From Sukarno to Megawati. Jadaypur Journal of International Relations, vol. 6(1), 29-44. Doi: 10.1177/0973598402110004. 
BвC Mundo. (2019, agosto 24). Por qué Papúa lleva 50 años reclamando su independencia de Indonesia. Disponible en https:/www.bbc.com/mundo/ noticias-internacional-49435967

Carr, A. (2014). Is Australia a middle power? A systemic impact approach, Australian Journal of International Affairs, vol. 68:1, 70-84, Doi: 10.1080/10357718.2013.840264.

Chandra, V. (2018). Rising Powers and The Future International Order. World Affairs: The Journal of International Issues, vol. 22(1), 10-23. Doi: $10.2307 / 48520045$.

Chapnick, A. (1999). The middle power, Canadian Foreign Policy Journal, 7:2, 73-82, Doi: 10.1080/11926422.1999.9673212.

Coleman, P.; et al. (2007). Arndt's Story: The life of an Australian economist. Canberra. ANU Press, 257-264 Retrieved from http://www.jstor.org/stable/j. ctt24h3vr.29

Connelly, A. (2014). Indonesia Foreign Policy Under President Jokowi. Lowy Institute for International Policy, 1-15, Retrieved from http://www.jstor.org/ stable/resrep 10156

Creswell, J. (2007). Qualitative Inquiry \& Research design. Choosing Among Five Approaches. California. Sage Publications.

Dirlik, A. (2007). Global South: Predicament and Promise. The Global South, vol.1(1), 12. Retrieved from http://www.jstor.org/stable/40339225

Efstathopoulos, C. (2018). Middle Powers and the Behavioural Model, Global Society, vol. 32:1, 47-69, Doi: 10.1080/13600826.2017.1351422.

Emmers, R. y Theo, S. (2018). Security Strategies of Middle Powers in the Asia Pacific. Melbourne: Melbourne University Press.

GK Today. (2017). IORA Leaders Summit held in Jakarta. Retrieved from https:/www.gktoday.in/currentaffairs/iora-leaders-summit-held-jakarta/
Heiduk, F. (2014). State disintegration and power politics in post-Suharto Indonesia. Third World Quarterly, vol.35:2, 300-315, Doi: 10.1080/01436597.2014.878491.

IORA International. (2017). Jakarta Concord. Retrieved from https:/www.iora.int/media/23875/jakartaconcord-7-march-2017.pdf

Iskandar, P. (2016). The Pancasila Delusion, Journal of Contemporary Asia, vol.46:4, 723-735, Doi: 10.1080/00472336.2016.1195430.

Kríž, Z.-Urbanovská, J.-Brajerčíková, S. (2019). The Middle Power Concept: Presenting a Complex Approach. In Politické Vedy, vol. 22, No. 4, 2019, pp.33-56, http://doi.org/10.24040/politickevedy.2019.22.4.33-56. ISSN 1335-2741.

Lee, Y. (2018) Maritime Merchandise Trade in Southeast Asia: Opportunities and Challenges, Maritime Affairs: Journal of the National Maritime Foundation of India, vol. 14:1, 51-69, Doi: 10.1080/09733159.2018.1482665.

Marks, D. van Zanden, J. (2012). An Economic History of Indonesia. 1800-2010. New York: Routledge Taylor \& Francis Group.

Mietzner, M. (2012). Indonesia: Yudhoyono's Legacy between Stability and Stagnation. Southeast Asian Affairs, 119-134. Retrieved from http://www.jstor. org/stable/41713990

Mietzner, M. (2015). Indonesia in 2014: Jokowi and the Repolarization of Post-Soeharto Politics. Southeast Asian Affairs 119-138. Retrieved from http://www. jstor.org/stable/44112801

Ministry of Foreign Affairs of the Republic of Indonesia. (2019). Indian Ocean Rim Association. Retrieved from https://kemlu.go.id/portal/en/read/167/halaman_list_lainnya/indian-ocean-rim-association Ministry of Foreign Affairs of the Republic of Indonesia. (2019). Foreign Policy: Foundation, Vision and Mission. Retrieved from https://kemlu.go.id/ 
portal/en/list/halaman_list_lainnya/10/landasan_visi_dan_misi_polugri

Monineath, E. (2013). The impact of the Ache and West Papua's separation in Indonesia. Royal University of Phnom Penh. Department of International Studies.

Nayanthara, R. (2016). Blue economy in Southeast Asia: Oceans as the new frontier of economic development, Maritime Affairs: Journal of the National Maritime Foundation of India, vol. 12:2, 1-15, Doi: 10.1080/09733159.2016.1244361.

Negara, S. (2016). Indonesia’s infrastructure development under the Jokowi administration. Southeast Asian Affairs, 145-166. Retrieved from https://www.jstor. org/stable/26466924

Nur, Y. (2019, October 15). Two Foreign Fishing Boats Caught Fishing Illegally in North Natuna Sea. Jakarta Globe. Retrieved from https://jakartaglobe. id/news/two-foreign-fishing-boats-caught-fishingillegally-in-north-natuna-sea/

Pavez \& Rosales. (2016). La proyección de Indonesia como potencia emergente. Revista Relaciones Internacionales, vol. 25(51). Retrieved from http:// sedici.unlp.edu.ar/handle/10915/57612

Sambhi, N. (2015). Jokowi's 'Global Maritime Axis': Smooth Sailing or Rocky Seas Ahead? Security Challenges, vol. 11(2), 39-56. Retrieved from https:// www.jstor.org/stable/26465437

Setiyanto, A. (2017). Strengthening Indonesia’s Role in Indian Ocean Through IORA - Universidad Pertahanan Indonesia - Jurnal Pertahanan, vol. 3, No. 1 .

Smith, A. (2003). Indonesia in 2002: Megawati's Way. Southeast Asian Affairs, 97-116. Retrieved from http://www.jstor.org/stable/27913229
Sputnik Mundo. (08/06/2018). Indonesia y Rusia firman contrato sobre los suministros de 11 aviones Sukhoi. Retrieved from https://mundo.sputniknews.com/ defensa/201806081079410383-indonesia-rusiaarmas-aviones-sukhoi/

Sulistiyanto, P. (2018). Indonesia in 2017: Jokowi's Supremacy and His Next Political Battles. Southeast Asian Affairs, 153-166. https://www.jstor.org/ stable/26492775

Syailendra, E. (2015). Papua Region under Jokowi: New President, New Strategies. S. Rajaratnam School of International Studies (RSIS), Nanyang Technological University. No. 110.

Thies, C. \& Sari, A. (2018). A Role Theory Approach to Middle Powers: Making Sense of Indonesia's Place in the International System. Contemporary Southeast Asia, vol. 40(3), 397-421. Retrieved from https:// www.jstor.org/stable/26545301.

Tiwon, S. (2000). From East Timor to Aceh: The disintegration of Indonesia? Bulletin of Concerned Asian Scholars, vol. 32:1-2, 97-104, Doi:10.1080/146 72715.2000 .10415792 .

Weatherbee, E. (2016). Trends in South Asia: Understanding Jokowi 's Foreign Policy. Singapore: ISEAS Iseas Yusof Ishak Institute.

Witular, R. (13/11/2014). Jokowi launches maritime doctrine to the world. The Jakarta Post. Retrieved from https://www.thejakartapost.com/ news/2014/11/13/jokowi-launches-maritimedoctrine-world.html

Zorlu, F. y Alam, S. (2019) Dispute in South Sea. Anadolu Agency. Retrieved from https://www. aa.com.tr/en/asia-pacific/dispute-in-south-chinasea- $/ 1566897$ 